\title{
The UT 19-channel DC SQUID based neuromagnetometer
}

\author{
H J M ter Brake, J Flokstra, W Jaszczukt, R Stammis, G K van Ancum, \\ A Martinezł and $\mathrm{H}$ Rogalla \\ University of Twente, Faculty of Applied Physics, PO Box 217, 7500 AE Enschede, The \\ Netherlands
}

\begin{abstract}
A 19-channel DC squid based neuromagnetometer is under construction at the University of Twente (UT). Except for the cryostat all elements of the system are developed at the UT. It comprises 19 wire-wound first-order gradiometers in a hexagonal configuration. The gradiometers are connected to planar DC SQUIDs fabricated with a $\mathrm{Nb} / \mathrm{Al}, \mathrm{AlO}_{x} / \mathrm{Nb}$ technology. For this connection we developed a method to bond a $\mathrm{Nb}$ wire to a $\mathrm{Nb}$ thin-film. The sQuids are placed in compartmentalised $\mathrm{Nb}$ modules. Further, external feedback is incorporated in order to eliminate cross talk between the gradiometers. The electronics basically consist of a phase-locked loop operating with a modulation frequency of $100 \mathrm{kHz}$. Between soujD and preamplifier a small transformer is used to limit the noise contribution of the preamplifier. In the paper the overall system is described, and special attention is paid to the souID module (bonding, compartments, external-feedback setup, output transformer).
\end{abstract}

\section{Introduction}

During the last few years biomagnetic instrumentation has changed from singlechannel SQUID systems to multichannel magnetometer units. In this way the magnetic field distribution around a subjects's body can be measured much faster and more reliably. Furthermore, spontaneous activity in the body can now be studied. Multichannel systems of about 20 to 30 channels are developed by or are under construction in university groups, for instance in Helsinki and Rome, at the PTB in Berlin and in industries like BTi, Siemens and Philips.

At the University of Twente a 19-channel DC SQUID magnetometer for brain research is under construction. In this paper several aspects of the system are described. First, the sensing-coil unit will be considered, that has been optimised with respect to the signal-to-noise ratio of the overall system. Then, attention is paid to the SQUID module in which other topics such as cross talk elimination by means of external feedback and bonding of a niobium wire to a niobium thin-film pad are involved. Finally, the electronics are shortly described.

\section{Sensing-coil unit}

First-order gradiometers are used as the sensing coils. Second-order gradiometers are not required, because our biomagnetism laboratory is on a low-noise location and

† On leave from: Institute of Low Temperature and Structure Research, Polish Academy of Sciences, PO Box 937, 50.950 Wroclaw, Poland.

‡ University of Zaragoza, C P S Department of Electrical Engineering and Informatics, Maria de Luna 3, 50015 Zaragoza, Spain. 
furthermore it is equipped with a magnetically shielded room. The gradiometers are optimised with respect to the overall signal-to-noise ratio of the magnetometer. In this optimisation the signal source is taken as a current dipole in a homogeneous conducting sphere having a diameter of $22 \mathrm{~cm}$. Source depths of 15 to $40 \mathrm{~mm}$ (measured from the skin) were considered. The resulting design is: coil diameter $20 \mathrm{~mm}$, baseline length $40 \mathrm{~mm}, 2$ coils of 3 turns each, with the turns separated by $0.5 \mathrm{~mm}$ in the proximal coil and by $6 \mathrm{~mm}$ in the compensating coil. Details of the gradiometer-optimisation method will be presented elsewhere.

The gradiometers are distributed in a hexagonal configuration over the bottom of a CTF-SST 140 cryostat. This bottom is concavely shaped having a curvature with a radius of $125 \mathrm{~mm}$. The sensing-coil unit is depicted in figure 1. As can be seen in this figure, the proximal coils are mounted side by side. The assembly is constructed of tufnol, a textile/epoxy composite. The wire used for the gradiometers is Formvar-insulated single-core niobium wire with a diameter of $0.1 \mathrm{~mm}$ (manufactured by California Fine Wire $\mathrm{Co}$ ). The calculated self-inductance of the gradiometers is $0.73 \mu \mathrm{H}$.

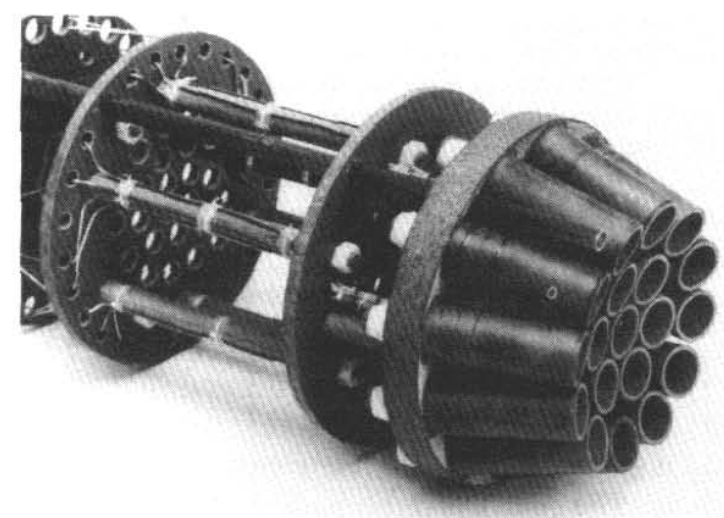

Figure 1. The 19-channel sensing-coil unit.

The calculated cross talk between two neighbouring gradiometers is $5 \%$. In the hexagonal configuration a number of gradiometers each couple to six neighbours (i.e. a hypothetical cross talk of up to $30 \%$ ). Therefore, we apply external feedback of the output of the SQUID systems to the flux-transformer circuits (ter Brake et al 1986). The realisation of this feedback is discussed in section 3 .

As mentioned earlier our laboratory is on a low-noise location at the University and equipped with a magnetically shielded room. Our room has only one $\mu$-metal wall and therefore suppresses low-frequency external fields by a factor of about 10 . By means of an active compensation technique we increased this low-frequency shielding factor to about 1000 (ter Brake et al 1990). To reduce noise further, electronic noise cancellation can be applied (ter Brake et al 1989). For this purpose three orthogonal $1 \mathrm{~cm}$ loops, connected to SQUID systems, are installed as references for three orthogonal components of the ambient magnetic ficld. This reference-coil unit can be seen in figure 2. As an additional noise reference a first-order gradiometer may be incorporated. All in all the neuromagnetometer therefore comprises 22 to 23 SQUID systems. 


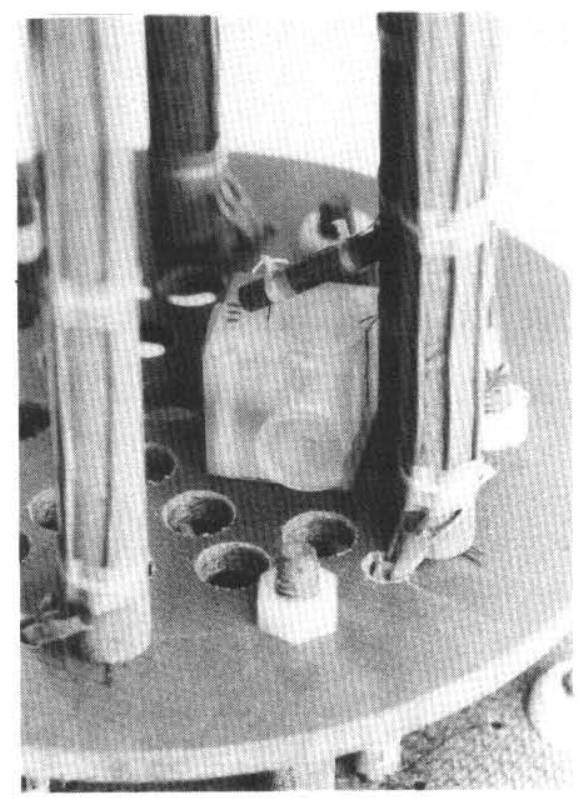

Figure 2. Detail of the sensing-coil unit, showing the noise-reference coils.

\section{DC SQUID module}

The DC SQUIDs are fabricated based on the $\mathrm{Nb} / \mathrm{Al}$ technology. Apart from standard Tesche-Clarke type sQUIDS, resistively and inductively shunted DC SQUIDS were also made and characterised (Houwman et al 1991). Based on the experiments performed with these SQUIDS, two configurations were selected, slightly optimised and fabricated in sufficiently large quantities to realise the multichannel neuromagnetometer. The first configuration is a Tesche-Clarke type DC SQUID with a SQUID inductance of 270 $\mathrm{pH}$, an input inductance of $0.1 \mu \mathrm{H}$, and a coupling factor of 0.8 . The forward transfer is typically $70 \mu \mathrm{V} \Phi_{0}^{-1}$. The other configuration is a resistively-shunted type with respectively the following specifications: $85 \mathrm{pH}, 0.1 \mu \mathrm{H}, 0.8$ and $110 \mu \mathrm{V} \Phi_{0}^{-1}$. The noise has not been measured in an optimal situation (i.e. significant noise contributions originate from the environment). Nevertheless, for both SQUIDS the white-noise spectral density is below $10^{-5} \Phi_{0} \mathrm{~Hz}^{-1 / 2}$. This noise level of the SQUID system is equivalent to an input noise of $5 \mathrm{fT} \mathrm{Hz}^{-1 / 2}$. The DC SQUID development at the University of Twente is considered in more detail in a separate contribution to this supplement (Flokstra et al 1991).

Each SQUID is placed on a solid niobium body that fits into a cylindrical lead shield. This module is shown in figure 3. The module is divided into compartments as depicted in figure 4 , in order to maximise the shielding of the different sections. The module compartments will now be considered separately. The gradiometer wires are connected to the SQUID input coil via niobium blocks with screws (compartment 1 in figure 4). Because the secondary coil of the external-feedback inductor has to be included in the flux-transformer circuit, three blocks are required. The blocks are insulated from the niobium body with epoxy. The design of compartment 1 with 
respect to its dimensions is based on a paper by Rigby et al (1990). The resulting worst-case imbalance contribution due to the loop formed by the wires on the screw blocks is less than $2 \times 10^{-5}$.

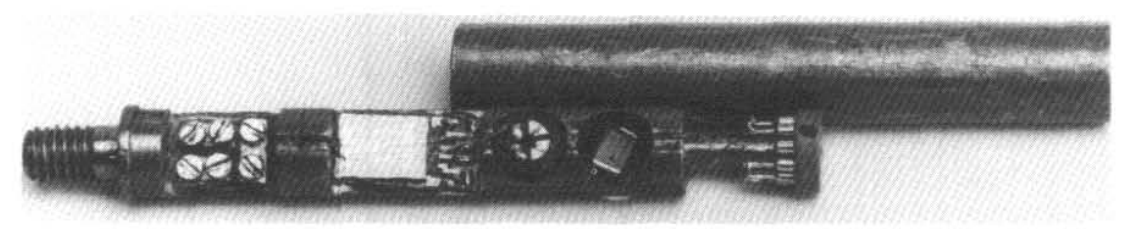

Figure 3. The sQuID module and lead shield.

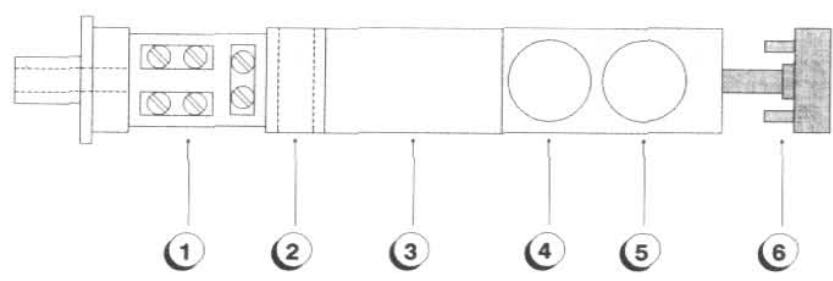

Figure 4. Compartments of the SQUID module: 1, screw connections for sensing coil and feedback inductor; 2 , hole for feedback inductor; 3 , plane for soUID chip; 4 , hole for output transformer, 5, hole for capacitor (tuning the transformer); and 6 , onnector.

As mentioned earlier external feedback is incorporated to eliminate gradiometer cross talk (ter Brake et al 1986). The feedback setup was designed with a number of constraints. First, the feedback circuit should not significantly contribute to the noise of the magnetometer. Second, the transfer of the system should be $1 \mathrm{~V}$ at the output corresponding to $1 \Phi_{0}$ in the SQUID. Finally, the secondary inductance of the feedback transformer should be small compared with that of the gradiometer. The transformer, placed in compartment 2, has a length of $4 \mathrm{~mm}$ and an outer diameter of $2.6 \mathrm{~mm}$. Its primary coil has about 90 turns and its secondary has 3 turns. The calculated mutual inductance inside the module enclosure is $0.3 \mu \mathrm{H}$, whereas the calculated secondary inductance is only about $0.02 \mu \mathrm{H}$. The primary coil is wound with niobium wire because copper wire would have resulted in a noise contribution of $7.5 \times 10^{-5} \Phi_{0} \mathrm{~Hz}^{-1 / 2}$ in the SQUID.

The DC SQUID chip is first glued onto a printed-circuit board. Then the board with the SQUID is glued onto the niobium body in compartment 3 . The glue that is used is low-temperature varnish manufactured by General Electrics. The board has bonding pads for bias, modulation and output-voltage connections. Special attention is paid to the input circuit, i.e. the connection of the screw blocks in section 1 to the SQUID input-coil. For this purpose we developed a method for bonding a niobium wire to a niobium thin-film pad. The method is characterised by two steps. First, the hardness of the niobium wire is reduced by a heat treatment. The wire is annealed in a vacuum chamber at a temperature of roughly $2200{ }^{\circ} \mathrm{C}$ for about $5 \mathrm{~min}$. Second, the niobium pad is covered with a thin layer of palladium $(4 \mathrm{~nm})$ to prevent it from oxidising. Superconducting bonds were realised using a straightforward ultrasonic bonding technique. The bonds and also the screw connections were tested by coupling 
a current to the flux-transformer circuit via the external feedback transformer. We measured superconductivity to a sensitivity level of $7 \times 10^{-14} \Omega$. Details on the bonding method and the test experiments will be presented elsewhere (Jaszczuk et al 1991).

A worst case resistance of $7 \times 10^{-14} \Omega$ in the flux-transformer circuit results in a thermal-noise contribution that decreases linearly with increasing frequency. In our situation the resulting worst-case noise flux spectral density in the SQUID is $2 \times 10^{-6} \Phi_{0} \mathrm{~Hz}^{-1 / 2}$ at $1 \mathrm{~Hz}$. Our DC souids have a white-noise level of typically $4 \times 10^{-6} \Phi_{0} \mathrm{~Hz}^{-1 / 2}$ and a $1 / f$ corner point at about $1 \mathrm{~Hz}$ (Flokstra et al 1991). Therefore, the thermal noise contribution of the bonds is below the intrinsic noise of the SQUID system.

In order to reduce the effect of preamplifier noise, a small output transformer is used between the SQUID and the preamplifier. The transformer is placed in compartment 4 of the module and is made of $0.09 \mathrm{~mm}$ diameter copper wire. It has a length of $5 \mathrm{~mm}$ and an outer diameter of $5.3 \mathrm{~mm}$, thus fitting in the $7 \mathrm{~mm}$ hole of compartment 4. The primary coil has 88 turns, and the secondary has 616 turns. The measured inductances are respectively $16(32)$ and $550 \mu \mathrm{H}(738 \mu \mathrm{H})$ whereas the coupling factor appeared to be $0.75(0.79)$. The values between brackets were measured outside the superconducting enclosure.

The output transformer is tuned to the modulation frequency of $100 \mathrm{kHz}$ via a capacative shunt of $10 \mathrm{nF}$ placed in compartment 5 . The gain of this tuned transformer is typically 10 at $100 \mathrm{kHz}$ with a moderate $Q$ of about 2 . The preamplifier noise is $1 \mathrm{nV} \mathrm{Hz}{ }^{-1 / 2}$ and thus the equivalent noise in the SQUID is $1.4 \times 10^{-6} \Phi \mathrm{Hz}^{-1 / 2}$ taking into account a forward transfer of $70 \mu \mathrm{V} / \Phi_{0}$. This means an equivalent effective field noise bclow $1 \mathrm{fT} \mathrm{Hz} z^{-1 / 2}$ which is very acceptable.

\section{Electronics}

All leads from the SQUID module to the electronics are soldered to the connector indicated in figure 4. From the connector to the top of the cryostat, the connections for bias, modulation and external feedback are made with twisted non-magnetic phosphor bronze wire having a diameter of $64 \mu \mathrm{m}$. This Formvar-insulated wire has a resistance of roughly $30 \Omega \mathrm{m}^{-1}$ and is manufactured by Lake Shore Cryotronics (wire type NM-42). The souid output lines are miniature coaxial cables from the same manufacturer (cable type $\mathrm{C}$ ). The preamplifier section is placed directly on top of the cryostat in an aluminium enclosure. It consists of Toshiba FETS (2SK146) in a cascode configuration, loaded with an $R C L$ network resonating at $100 \mathrm{kHz}$. The quality factor is about 3.5 and the preamplifier gain is typically 400 .

The control and detection electronics is realised in a miniaturised version on a single Eurocard for each channel. Because of the miniaturisation and the fact that the system is not designed to be a general purpose magnetometer, several parameters have been fixed among which are the sensitivity and the frequency bandwidth $(5 \mathrm{kHz})$. The sensitivity is $1 \mathrm{~V}$ at the output for $1 \Phi_{0}$ in the SQulD. The dynamic range of $10 \mathrm{~V}$ corresponds to an effective field of about $0.5 \mathrm{nT}$ in the gradiometer. The power supplies and the bias-current supplies are independent for each channel, whereas the $100 \mathrm{kHz}$ modulation for all channels is obtained from an external oscillator. The electronics have been developed independently from the DC SQUID devices. System 
parameters such as dynamic behaviour, the gain of the feedback path, and the openloop gain have been adjusted by means of a DC SQUID simulator (Martinez et al 1990).

Before analogue-to-digital conversion some analogue filtering is necessary. For this purpose we developed a $50 \mathrm{~Hz}$ adaptive notch filter that suppresses the mains frequency by more than $60 \mathrm{~dB}$ with a bandwidth of only $1 \mathrm{~Hz}$. Further, high-pass and low-pass filters will be applied, the latter also functioning as anti-alias filters for the $1 \mathrm{kHz}$ sampling frequency. Thus signals can be measured without significant phase shift up to a frequency of $100 \mathrm{~Hz}$, which is adequate for neuromagnetic experiments.

\section{Conclusion}

We presented the design of our 19-channel DC SQUiD based neuromagnetometer. Various aspects concerning the sensing-coil unit, the SQUID module and the electronics are discussed. We are presently testing a three-channel prototype especially focusing on problems with respect to interference. After the successful operation of this prototype the 19-channel system will be completed and installed in the magnetically shielded room of the Biomagnetic Centre Twente. It will be used among other applications for studies on epilepsy and cognitive processes.

\section{References}

Flokstra J, Adelerhof D J, Houwman E P, Ve!dhuis D and Rogalla H 1991 Josephson junctions and DC soujds based on $\mathrm{Nb} / \mathrm{Al}$ technology This volume pp $59-66$

Houwman E P, Veldhuis D, Flokstra J, ter Brake H J M, Jaszczuk W, Martinez, A and Rogalla H 1991 DC SOUtD sensor system for multichannel neuromagnetometry IEEE Trans. Magn. 27 2955-8

Jaszczuk W, ter Brake H J M, Flokstra J, Veldhuis D, Stammis R and Rogalla H 1991 Bonding of a niobium wire to a niobium thin-film Meas. Sci. Techn. 2 1121-2

Martinez A, Flokstra J, Rillo C, Angurel L A, Garcia L M and ter Brake H J M 1990 A low-noise souID simulator with a large dynamic mange of up to eight flux quanta Cryogenics 30 324-9

Rigly K W, Marek D and Chui T C. P 1990 squid holder with high magnetic shielding Rev. Sci. hustrum. $61834-8$

ter Brake H J M, Dunajski Z, Van der Mheen W A G and Flokstra J 1989 Electronic balancing of multichannel sould magnetometers J. Phys. E. Sci. Instrum. 22 560-4

ter Brake H J M, Feuren F H, Ulfman J A and Flokstra J 1986 Elimination of flux-transformer cross talk in multichannel sould magnetometcrs Cyogentics $26667-70$

ter Brake H J M, Wieringa H J anú Rogalla $H 1991$ Improvement of the performance of a $f$-metal magnetically shielded room by means of active compensation Meas. Sci. Tech. 2 596-601 